Internat. J. Math. \& Math. Sci.

Vol. 24, No. 4 (2000) 231-235

S0161171200004191

(c) Hindawi Publishing Corp.

\title{
A NOTE ON KAKUTANI TYPE FIXED POINT THEOREMS
}

\author{
A. R. KHAN, N. HUSSAIN, and L. A. KHAN
}

(Received 3 September 1998 and in revised form 29 November 1999)

\begin{abstract}
We present Kakutani type fixed point theorems for certain semigroups of self maps by relaxing conditions on the underlying set, family of self maps, and the mappings themselves in a locally convex space setting.

Keywords and phrases. Fixed point, semigroup of self maps, locally convex space, nonexpansive map.
\end{abstract}

2000 Mathematics Subject Classification. Primary 41A65, 46A03, 47H10.

1. Introduction. Using a technique of Tarafdar [9], we establish fixed point theorems by utilizing following semigroups under composition of self maps $T$ on a subset $M$ of a Hausdorff locally convex space

(i) $\mathscr{F}=C_{T}=\{f: M \rightarrow M \mid f T=T f\}$,

(ii) $\mathscr{F}=\left\{T^{n}: n \in \mathbb{N} \cup\{0\}\right\}$,

(iii) $\mathscr{F}=$ identity map.

In the sequel $(E, \tau)$ will be a Hausdorff locally convex topological vector space. A family $\left\{p_{\alpha}: \alpha \in I\right\}$ of seminorms defined on $E$ is said to be an associated family of seminorms for $\tau$ if the family $\{r U: r>0\}$, where $U=\bigcap_{i=1}^{n} U_{\alpha_{i}}$ and $U_{\alpha_{i}}=\left\{x: p_{\alpha_{i}}(x)<\right.$ $1\}$, forms a base of neighbourhoods of zero for $\tau$. A family $\left\{p_{\alpha}: \alpha \in I\right\}$ of seminorms defined on $E$ is called an augmented associated family for $\tau$ if $\left\{p_{\alpha}: \alpha \in I\right\}$ is an associated family with the property that the seminorm $\max \left\{p_{\alpha}, p_{\beta}\right\} \in\left\{p_{\alpha}: \alpha \in I\right\}$ for any $\alpha, \beta \in I$. The associated and augmented associated families of seminorms shall be denoted by $A(\tau)$ and $A^{*}(\tau)$, respectively. It is well known that given a locally convex space $(E, \tau)$, there always exists a family $\left\{p_{\alpha}: \alpha \in I\right\}$ of seminorms defined on $E$ such that $\left\{p_{\alpha}: \alpha \in I\right\}=A^{*}(\tau)$ (see [7, page 203]).

The following construction will be crucial. Suppose that $M$ is a $\tau$-bounded subset of $E$. For this set $M$ we can select a number $\lambda_{\alpha}>0$ for each $\alpha \in I$ such that $M \subset \lambda_{\alpha} U_{\alpha}$, where $U_{\alpha}=\left\{x: p_{\alpha}(x) \leq 1\right\}$. Clearly, $B=\bigcap_{\alpha} \lambda_{\alpha} U_{\alpha}$ is $\tau$-bounded, $\tau$-closed, absolutely convex, and contains $M$. The linear span $E_{B}$ of $B$ in $E$ is $\bigcup_{n=1}^{\infty} n B$. The Minkowski functional of $B$ is a norm $\|\cdot\|_{B}$ on $E_{B}$. Thus $\left(E_{B},\|\cdot\|_{B}\right)$ is a normed space with $B$ as its closed unit ball and $\sup _{\alpha} p_{\alpha}\left(x / \lambda_{\alpha}\right)=\|x\|_{B}$ for each $x \in E_{B}$.

A self map $T$ on $M$ is said to be

(i) $A^{*}(\tau)$-nonexpansive if for all $x, y \in M$,

$$
p_{\alpha}(T x-T y) \leq p_{\alpha}(x-y) \text { for each } p_{\alpha} \in A^{*}(\tau) \text {. }
$$

(ii) $A^{*}(\tau)$-asymptotically nonexpansive if for each $x, y \in M$, 


$$
p_{\alpha}\left(T^{n} x-T^{n} y\right) \leq k_{n} p_{\alpha}(x-y), \quad n=1,2,3, \ldots, \text { for each } p_{\alpha} \in A^{*}(\tau),
$$

where $\left\{k_{n}\right\}$ is a fixed sequence of real numbers such that $k_{n} \rightarrow 1$ as $n \rightarrow \infty$.

In sequel, for simplicity, we shall call $A^{*}(\tau)$-nonexpansive $\left(A^{*}(\tau)\right.$-asymptotically nonexpansive) maps to be nonexpansive (asymptotically nonexpansive).

Common fixed points of nonexpansive maps and best approximations have been considered in normed spaces (see [1, 3]). We prove common fixed point theorems for asymptotically nonexpansive maps in the setting of a locally convex space.

\section{Results}

LEMMA 2.1. Let $M$ be a $\tau$-bounded subset of a Hausdorff locally convex space $(E, \tau)$ and $T: M \rightarrow M$ be asymptotically nonexpansive map. Then $T$ is asymptotically nonexpansive on $M$ with respect to $\|\cdot\|_{B}$.

Proof. By hypothesis for $x, y \in M$ and $n=1,2,3, \ldots$,

$$
p_{\alpha}\left(T^{n} x-T^{n} y\right) \leq k_{n} p_{\alpha}(x-y) \text { for each } p_{\alpha} \in A^{*}(\tau),
$$

where $\left\{k_{n}\right\}$ is a real sequence converging to 1 ,

$$
\begin{aligned}
\sup _{\alpha} p_{\alpha}\left(\frac{T^{n} x-T^{n} y}{\lambda_{\alpha}}\right) & \leq k_{n} \sup _{\alpha} p_{\alpha}\left(\frac{x-y}{\lambda_{\alpha}}\right), \\
\left\|T^{n} x-T^{n} y\right\|_{B} & \leq k_{n}\|x-y\|_{B},
\end{aligned}
$$

where $\left\{k_{n}\right\} \rightarrow 1$ as $n \rightarrow \infty$ and is a fixed real sequence. This completes the proof.

Note that $\left(E_{B}, \tau\right) \subset\left(E_{B},\|\cdot\|_{B}\right)$ so a set compact in $\left(E_{B}, \tau\right)$ need not be compact in $\left(E_{B},\|\cdot\|_{B}\right.$ ) (cf. [8, page 159, problem 3(c)]). To overcome this difficulty we use finite dimensionality to obtain following generalization of [9, Theorem 2.1].

THEOREM 2.2. Let $M$ be a nonempty convex $\tau$-bounded, $\tau$-complete finite dimensional subset of a Hausdorff locally convex space $(E, \tau)$. Suppose $\mathscr{F}$ is a commutative semigroup of asymptotically nonexpansive self maps of $M$. Then there exists a point $a \in M$ such that

$$
T(a)=a \quad \text { for all } T \in \mathscr{F} .
$$

Proof. Since $M$ is $\tau$-complete, it follows that $\left(E_{B},\|\cdot\|_{B}\right)$ is a Banach space and $M$ is complete in it. A closed, bounded and finite dimensional subset of a normed space is compact by [2, Theorem on page 10 ] so $M$ is compact in $\left(E_{B},\|\cdot\|_{B}\right)$. By Lemma 2.1, each $T \in \mathscr{F}_{F}$ is $\|\cdot\|_{B}$-asymptotically nonexpansive. Hence $\mathscr{F}$ is a commutative semigroup of asymptotically nonexpansive self maps of a compact convex subset $M$ of the Banach space $\left(E_{B},\|\cdot\|_{B}\right)$. The family $\mathscr{F}$ has a common fixed point by [4, Theorem 3.1].

We now prove another fixed point theorem for locally convex spaces by making use of Jungck and Sessa [6, Theorem 3]; see also [1, Corollary 2.3] and [5, Theorem 1].

THEOREM 2.3. Let $M$ be a $\tau$-bounded, $\tau$-sequentially closed and finite dimensional subset of a Hausdorff locally convex space $(E, \tau)$. Suppose that $M$ is starshaped with 
starcentre $q \in M$ and $T: M \rightarrow M$ is nonexpansive. Let $\mathscr{F}$ be a family of affine nonexpansive self maps of $M$ commuting with $T$ and leaving $q$ fixed. Suppose for each pair $(x, y) \in M^{2}$, there exists $f=f(x, y)$ and $g=g(x, y)$ in $\mathscr{F}$ such that

$$
p_{\alpha}(T x-T y) \leq p_{\alpha}(f x-g y) \quad \text { for all } p_{\alpha} \in A^{*}(\tau)
$$

Then there exists $a \in M$ such that

$$
a=T(a)=h(a) \quad \text { for all } h \in \mathscr{F} \text {. }
$$

Proof. Since $\|\cdot\|_{B}$-topology is finer than the relative $\tau$-topology on $E_{B},\|\cdot\|_{B^{-}}$ $\operatorname{cl}(M) \subset \tau$-sequential-cl $(M)=M$. Therefore, $M$ is $\|\cdot\|_{B}$-closed in the normed space $\left(E_{B},\|\cdot\|_{B}\right)$. As above, $M$ is a compact subset of $\left(E_{B},\|\cdot\|_{B}\right)$. Moreover, $T$ and each $h \in \mathscr{F}_{F}$ is nonexpansive in $(E, \tau)$, which by Lemma 2.1 implies that $T$ and each $h \in \mathscr{F}$ is $\|\cdot\|_{B^{-}}$ nonexpansive-so certainly $\|\cdot\|_{B}$-continuous. And from (2.4) we obtain for $x, y \in M$,

$$
\sup _{\alpha} p_{\alpha}\left(\frac{T x-T y}{\lambda_{\alpha}}\right) \leq \sup _{\alpha} p_{\alpha}\left(\frac{f x-g y}{\lambda_{\alpha}}\right) .
$$

Thus

$$
\|T x-T y\|_{B} \leq\|f x-g y\|_{B} \text { for } x, y \in M \text {. }
$$

A comparison of our hypothesis with that of [6, Theorem 3] tells us that we can now apply [6, Theorem 3] to $M$ as a subset of $\left(E_{B},\|\cdot\|_{B}\right)$ to conclude that there exists $a \in M$ such that $a=T(a)=h(a)$ for all $h \in \mathscr{F}$.

COROLLARY 2.4. Let $M$ be a $\tau$-bounded, $\tau$-sequentially closed, and finite dimensional subset of a Hausdorff locally convex space $(E, \tau)$. Assume $M$ is starshaped with starcentre $q \in M$. Suppose $T, I: M \rightarrow M$ are nonexpansive, $I$ is affine and leaving $q$ fixed and $T I=I T$. Suppose for $x, y \in M$, there exist $n=n(x, y), m=m(x, y)$ in $\mathbb{N}_{0}=\{0,1,2, \ldots\}$ such that

$$
p_{\alpha}(T x-T y) \leq p_{\alpha}\left(I^{m} x-I^{n} y\right) \quad \text { for each } p_{\alpha} \in A^{*}(\tau)
$$

Then $T$ and I have a common fixed point.

Proof. Let $\mathscr{F}=\left\{I^{n}: n \in \mathbb{N}_{0}\right\}\left(I^{0} x=x\right)$. For each $n, I^{n}$ is affine, $T I^{n}=I^{n} T$ and $I^{n}: M \rightarrow M$ since $I$ has these properties. Further (2.8) assures that $\mathscr{F}$ and its members satisfy (2.4) and the hypotheses of Theorem 2.3; consequently, the conclusion of the corollary follows.

COROLLARY 2.5. Let $M$ be a $\tau$-bounded, $\tau$-closed finite dimensional starshaped subset of a Hausdorff locally convex space $(E, \tau)$ and $T$ a nonexpansive self map of $M$. Then $T$ has a fixed point.

Finally, we consider an application of Corollary 2.4 to best approximation theory. A related result for normed spaces was given in [6, Theorem 4]. For any $\bar{x} \in E, C \subseteq E$ 
and $p_{\alpha} \in A^{*}(\tau)$, let

$$
d_{p_{\alpha}}(\bar{x}, C)=\inf \left\{p_{\alpha}(y-\bar{x}): y \in C\right\}
$$

and let

$$
D=\left\{y \in C: p_{\alpha}(y-\bar{x})=d_{p_{\alpha}}(\bar{x}, C) \text { for all } p_{\alpha} \in A^{*}(\tau)\right\}
$$

THEOREM 2.6. Let $T$ and $I$ be self maps of a Hausdorff locally convex space $(E, \tau)$ and let $C \subseteq E$ be such that $T: \partial C \rightarrow C$. Let $T$ and I leave $\bar{x} \in E$ fixed and satisfy (2.8) for all $x, y \in D \cup\{\bar{x}\}$. Suppose $I$ is nonexpansive and affine, $T$ is nonexpansive on $D$, $I T=T I$ on $D$, and $D$ is nonempty $\tau$-bounded, $\tau$-sequentially closed, finite dimensional and starshaped with respect to q. If I leaves $q$ invariant and $I(D) \subseteq D$, then there exists $a \in D$ such that $a=I(a)=T(a)$.

Proof. Let $y \in D$. Then $I^{n} y \in D$ for $n \in \mathbb{N}_{0}$ since $I(D) \subseteq D$. By definition of $D$, $y \in \partial C$ and since $T: \partial C \rightarrow C$, it follows that $T y \in C$. By (2.8), for each $p_{\alpha} \in A^{*}(\tau)$,

$$
p_{\alpha}(T y-\bar{x})=p_{\alpha}(T y-T \bar{x}) \leq p_{\alpha}\left(I^{n} y-I^{m} \bar{x}\right)
$$

for some $n, m \in \mathbb{N}_{0}$. As $I^{m} \bar{x}=\bar{x}$, we get

$$
p_{\alpha}(T y-\bar{x}) \leq p_{\alpha}\left(I^{n} y-\bar{x}\right) \text { for all } p_{\alpha} \in A^{*}(\tau) .
$$

Again since $T y \in C$ and $I^{n} y \in D$, the definition of $D$ further implies that $T y \in D$. Consequently, $T, I: D \rightarrow D$ and the conditions of Corollary 2.4 are satisfied. Hence there exists $a \in D$ such that $a=I(a)=T(a)$.

ACKNOWLEDGEMENTS. The first author acknowledges gratefully the support provided by the King Fahd University of Petroleum and Minerals during this research. The authors thank Naseer Shahzad for useful discussions and the referee for pointing out a short proof of Theorem 2.3.

\section{REFERENCES}

[1] M. A. Al-Thagafi, Common fixed points and best approximation, J. Approx. Theory 85 (1996), no. 3, 318-323. MR 97f:47051. Zbl 858.41022.

[2] E. W. Cheney, Introduction to Approximation Theory, McGraw-Hill Book Co., New York, Toronto, Ont., London, 1966. MR 36\#5568. Zbl 161.25202.

[3] R. DeMarr, Common fixed points for commuting contraction mappings, Pacific J. Math. 13 (1963), 1139-1141. MR 28\#2446. Zbl 191.14901.

[4] K. Goebel, W. A. Kirk, and R. L. Thele, Uniformly Lipschitzian families of transformations in Banach spaces, Canad. J. Math. 26 (1974), 1245-1256. MR 50\#10919. Zbl 285.47039.

[5] J. R. Jachymski, Another proof of the Markov-Kakutani theorem, and an extension, Math. Japon. 47 (1998), no. 1, 19-20. MR 99f:47078. Zbl 930.47027.

[6] G. Jungck and S. Sessa, Fixed point theorems in best approximation theory, Math. Japon. 42 (1995), no. 2, 249-252. MR 96f:47112. Zbl 834.54026.

[7] G. Köthe, Topological Vector Spaces. I, Springer-Verlag New York Inc., New York, 1969. MR 40\#1750.

[8] H. L. Royden, Real Analysis, The Macmillan Co., New York, Collier-Macmillan Ltd., London, 1963. MR 27\#1540. Zbl 121.05501. 
[9] E. Tarafdar, Some fixed-point theorems on locally convex linear topological spaces, Bull. Austral. Math. Soc. 13 (1975), no. 2, 241-254. MR 53\#8979. Zbl 318.47032.

A. R. Khan: BaHAUdDin ZaKariYa University, Multan 60800, PAKISTAN

Current address: Department of MATHEMATICAl SCIENCES, King FAHD University of Petroleum AND Minerals, DHAHRAn, 31261, SAUdi ARABia

E-mail address: arahim@kfupm. edu . sa

N. Hussain: Centre for Advanced Studies in PuRe ANd Applied Mathematics, BaHAudDIN ZAKARIYA UNIVERSITY, MULTAN 60800, PAKISTAN

L. A. Khan: Department of Mathematics, King Abdul Aziz University, P.O. Box 9028, JEDDAH-21413, SAUDI ARABIA 


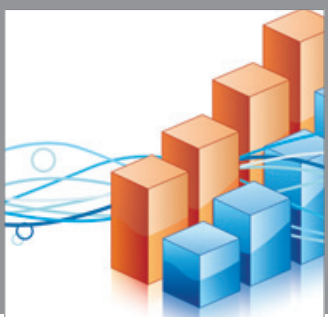

Advances in

Operations Research

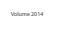

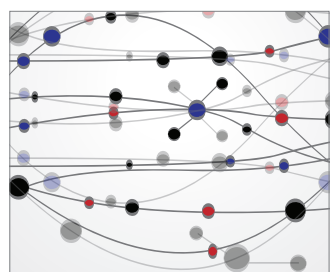

\section{The Scientific} World Journal
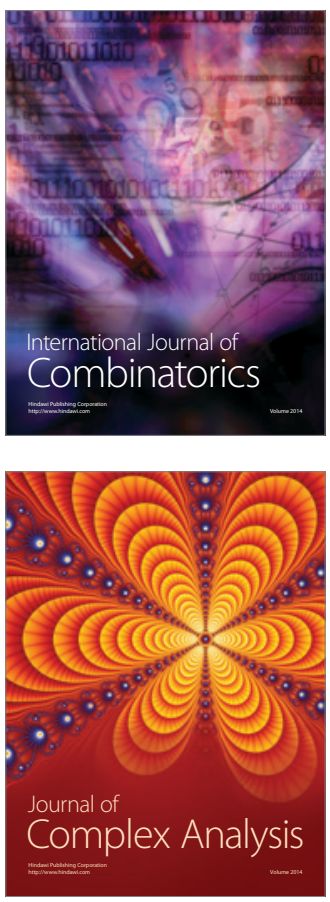

International Journal of

Mathematics and

Mathematical

Sciences
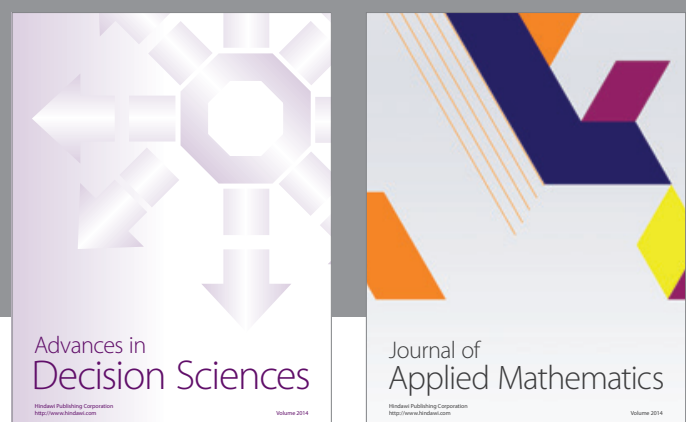

Journal of

Applied Mathematics
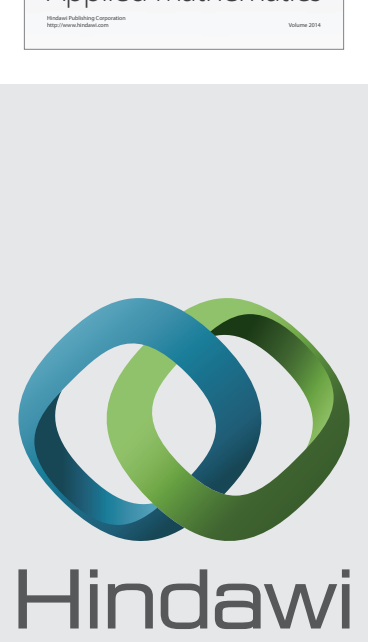

Submit your manuscripts at http://www.hindawi.com
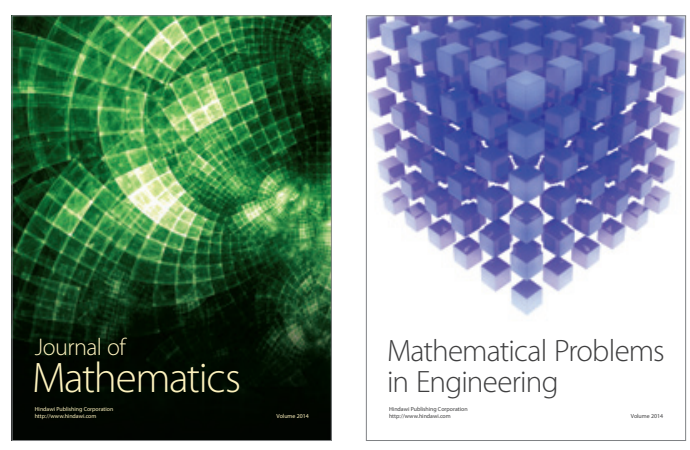

Mathematical Problems in Engineering
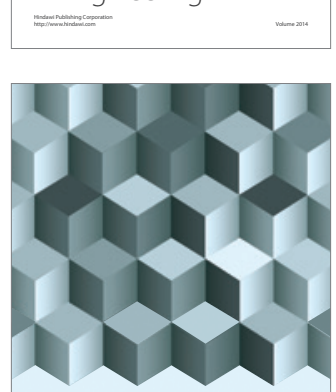

Journal of

Function Spaces
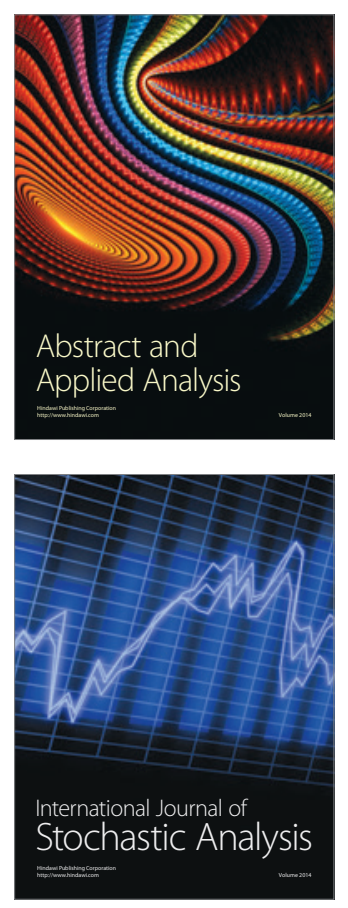

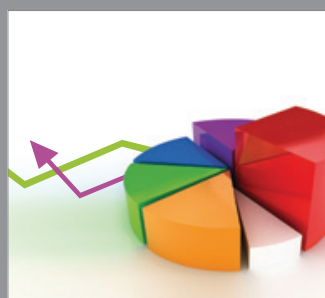

ournal of

Probability and Statistics

Promensencen
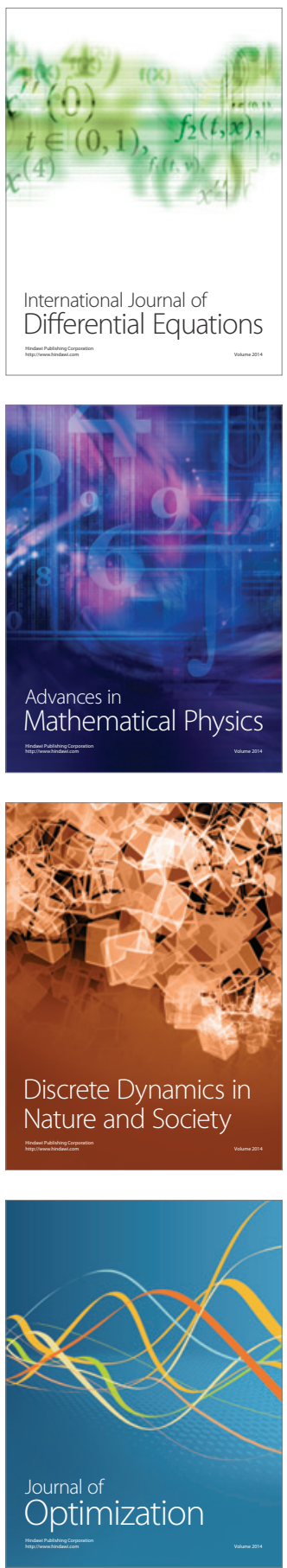